\title{
XLIII.
}

\section{Ueber die Einwirkung des Salmiaks auf Kupfer.}

\author{
Von
}

Dr. H. Ritthausen,

Assistenten am I. Universitätslaboratorium zu Leipzig.

Als Produkt der gegenseitigen Einwirkung von Salmiaklösung auf Kupfer bei Luftzutritt kennt man bis jetzt nur das Braunschweiger Grün $=(\mathrm{CuCl}, 3 \mathrm{CuO})+4 \mathrm{HO}$. Ich hielt es für wahrscheinlich, dass diese Verbindung nur ein secundäres Produkt sei. Um zu untersuchen, welche Verbindungen sich zunächst bei der Reaction bilden, stellte ich in eine kalt gesătligte Salmiaklüsung mehrere Kupferstreifen, so, dass diese noch über die Flüssigkeit hinaus ragten, und überliess das Ganze bei ungehindertem Luftzutritt in einem Becherglase bei gewöhnlicher Temperatur sich selbst. Nach einigen Stunden schon hatte sich die Salmiaklösung stark gebläut und am folgenden Tage zeigten sich auf der Oberfläche derselben kleine zu Sternen gruppirte grüne Krystallnadeln, übrigens aber liess sich in den daraul folgenden Tagen nichts Bemerkenswerthes wahrnehmen. Als ich nach Verlauf von ungefăhr zwei Monaten die Lösung wieder untersuchte, zeigte sie sich mit einer hellblauen Kruste bedeckt; auf dem Boden des Glases aber sah ich eine kleine Menge ziemlich grosser dunkelblauer Prismen, welche in noch grōsserer Anzahl an den Kupferstreifen sassen. Die Krystalle besassen nach dem Herausnehmen aus der Flüssigkeit viel Glanz, wurden aber nach dem Abtrocknen bald matt und bedeckten sich mit einer dünnen Journ. f. prakt. Chemie. LIX. 7. 
grünlichen Schicht. Eine vorlăufige qualitative Untersuchung ergab, dass sie eine grosse Menge Kupferoxydul enthielten, etwas Kupferoxyd, ferner Chlor und Ammoniak; von Wasser wurden sie zersetzt.

Da Wärme die Einwirkung von Salmiak auf Kupfer möglicherweise unterstützen konnte, kochte icl die schon benutzte Salmiaklōsung mit denselben Kupferstreifen, bis sich Ammoniak zu entwickeln begann. Die filtrirte Flüssigkeit schied während des Erkaltens zuerst kleine weisse glänzende Krystallkörner $a b$, nach diesen aber eine Menge der blauen Krystalle. Bei Wiederholung dieses Versuchs mit einer grǒssern Menge Kupfer und der von den Krystallen abgegnssenen Salmiaklösung wurde die Anfangs blaue Flüssigkeit vollkommen farblos, bläute sich aber sofort, als sie filtrirt wurde. Bald nach dem Filtriren bildeten sich zuerst die weissen, und bei weiterem Erkalten auch die blauen Krystalle in grosser Menge. Nach vollständigem Erkalten der Flüssigkeit zeigten sich darin fast erbsengrosse weisse, sehr glänzende, glashelle und vollkommen ausgebildete Rhombendodekaëder, die aber zum Theil mit blauen Krystallen durchwachsen waren. Aus der Flüssigkeit herausgenommen färbten sie sich sofort etwas violett und verloren den Glanz. Nach einer vorlăufigen Prüfung möglichst reiner weisser Krystalle, bestanden sie aus Kupferoxydul (ohne Kupferoxyd), Chlor und Ammoniak.

Die blaue, von den Krystallen abgegossene Flüssigkeit färbte sich sebr bald grün und schied dann auf der Oberfläche kleine würfelförmige Krystalle von prăchtig smaragdgrūner Farbe ab.

Die weitere Untersuchung dieser drei Körper ergab Folgendes.

Das weisse Salz. Dieses Salz ist, wie aus den weiter unten angeführten Analysen hervorgeht, eine Verbindung von Kupferchlorür und Ammoniak. Aus den bisher gegebenen allgemeinen Andeutungen über die Bildung der erwälmten drei Verbindungen geht schon deutlich hervor, dass dieses Salz nur dann entsteht, wenn die Salmiaklösung viel Kupfer aufgelöst hat. Man erhitzt eine kaltgesăttigte Salmiaklösung mit Kupfer (am besten eignen sich hierzu feine Kupferspähne oder durch Eisen aus Kupfervitriollösung gefâlltes Kupfer) bis nahe zum Sieden, so lange als keine starke Ammoniakentwickelung bemerkbar ist. Die Lösung wird, selbst wenn sie ganz farblos war, beim Fil- 
triren tief blau, oft bis zur Undurchsichtigkeit, und aus derselben scheiden sich, wenn sie sehr gesättigt ist, sofort kleine weisse Krystalle aus. Bei langsamer Abkühlung erhält man das Salz in grossen schön ausgebildeten Rhombendodekaëdern, gemengt mit Krystallen des blauen Salzes, von dem sie sich durch Auslesen trennen lassen. Die zuerst aus der noch heissen Lösung ausgeschiedenen kleinen Krystalle setzen sich rasch zu Boden; giesst man die überstehende Lósung, noch bevor sie blaue lirystalle abgesetzt hat, ab, so erhält man reines Salz, das durch wiederholtes Uebergiessen mit Alkohol von der Mutterlauge befreit werden kann. Selbst bei dem vorsichtigsten Trocknen 2 wischen Fliesspapier zersetzen sich diese kleinen Krystalle. etwas an der Luft, sie absorbiren Sauerstoff und bedecken sich mit einer sehr dünnen blāulich-grünen oxydhaltigen Schicht; die grōssern Krystalle werden an der Luft schnell etwas violett, doch ist diese Färbung nur ganz oberflächlich.

Am leichtesten erhält man das weisse Salz, wenn man die ursprüngliche siedende Lŏsung unmittelbar in die Hälfte ihres Volumens Wasser filtrirt; hierdurch wird der grösste Theil des blauen Salzes zersetzl, auch ein Theil des weissen, es scheidet sich gelbes Kupferoxydulbydrat aus; filtrirt man von Neuem, so scheiden sich aus der noch warmen Flüssigkeit beim Erkalten zablreiche weisse Krystalle von ziemlicher Grösse und ohne alle Beimengung aus; diese werden am besten mit Alkohol gewaschen und zwischen Fliesspapier rasch getrocknet, wobei indessen das Mattwerden derselben nicht vermieden werden kann.

Das Salz wird von Wasser zersetzt, es färbt sich gelb; wird es längere Zeit mit heissem Wasser ausgewaschen, so wird der Rückstand allmählich braun und man erhält dann Reaction auf Kupferoxyd. Die Waschwässer reagiren ammoniakalisch und enthalten Chlor, dessen Menge sich allmählich vermindert. In Säuren ist es ohne Zersetzung nicht löslich; es löst sich in concentrirter heisser Salmiaklosung, doch wird diese Lösung blau in Folge der Einwirkung des Sauerstoffs der Luft. Bei $100^{\circ}$ getrocknet verliert es selbst nach langer Zeit wenig an Gewicht, es verfüchtigt sich etwas Ammoniak und die Krystalle werden in Folge von Oxydation braun. Wird es stärker erhitzt, so entweicht nur Ammoniak, kein Salmiak, etwas Kupferchlorür und zurück bleibt ganz weisses Kupferchlorür. Das Salz enthält 
kein Krystallwasser. Durch Alkohol wird es nicht zersetzt. Zur Analyse wurde das Salz in Salzsäure mit Zusatz von etwas Salpetersāure gelöst, das Kupleroxyd durch Kali gefält. Zur Bestimmung des Chlors wurde es in reiner Salpetersäure gelöst. Das Ammoniak wurde aus salzsaurer Lósung mit Platinchlorid gefällt. Zu jeder Bestimmung wurde eine besondere Quantität Salz genommen.

Ich erbielt folgende Resultate:

\begin{tabular}{|c|c|c|c|c|c|c|c|}
\hline $\begin{array}{l}56 \\
97\end{array}$ & & & $\begin{array}{l}0,387 \\
0,6695\end{array}$ & $\mathrm{CuO}$ & $\begin{array}{l}=0,309 \\
=0,534\end{array}$ & & $\begin{array}{l}=54,59 \\
=54,79\end{array}$ \\
\hline 5075 & $\because$ & & 0,344 &, & $=0,274$ & "; & $=53,99$ \\
\hline, 6485 & , & & 0,438 & & $=0,350$ & & $=53,97$ \\
\hline 38 & $"$ & $"$ & 0,483 & ClAg & $=0,115$ & $\mathrm{Cl}$ & $=29,87$ \\
\hline 35 & $"$ & $"$ & 0,444 & 0 & $=0,110$ & & $=30,98$ \\
\hline 352 & $;$ & $"$ & 0,4338 & , & $=0,107$ & & $=30,4$ \\
\hline 6405 & $\eta$ & , & 0,5185 & $\mathbf{P t}$ & $=0,089$ & NH & $=13,895$ \\
\hline
\end{tabular}

Die Mittelzahlen dieser Resultate sind:

$$
\begin{aligned}
& \mathrm{Cu}=54,31 \text { p. C. } \\
& \mathrm{Cl}=30,38 \% \\
& \mathrm{NH}_{3}=13,895 "
\end{aligned}
$$

Daraus berechnet sich die Formel:

welche fordert:

$$
\mathrm{Cu}_{2} \mathrm{Cl}+\mathrm{NH}_{3} \text {. }
$$

$$
\begin{aligned}
& \mathrm{Cu}=54,7 \\
& \mathrm{Cl}=30,63 \\
& \mathrm{NH}_{3}=14,67
\end{aligned}
$$

Da die Krystalle oberflächlich immer etwas oxydirt sind, und eine Spur Ammoniak verloren haben, so werden sich daraus die geringen Differenzen erklären, welche einige Analysen ergeben.

Becquerel (Gmelin, Lehrb., 5te Aufl., III. 425) hat „auf galvanischem Wege durch Einwirkung von Kupfer auf Salmiaklösung theils Anfangs wasserhelle, dann amethystroth werdende Octaëder, theils sechsseilige Săulen, theils erst farblose, dann gelbe Irrystalle von abweichender Form erhalten, aus welchen Wasser den Salmiak auszieht". Dasselbe Salz erhielt er bei Einwirkung des Kupfers auf Salmiaklösung an der Luft, hielt es aber für Kupfercllorïrsalmiak. Nach den im Vorstehenden 
angeführten Reactionen des Kupferchlorür-Ammoniaks sind die Becquerel'schen Krystalle wahrscheinlich das von mir untersuchte Kupferchlorür-Ammoniak.

Dieses Salz bildet sich stets, wenn Kupfer im Ueberschuss bei Luftzutritt vorhanden ist; die letztere Bedingung ist zur Zersetzung des Salmiaks durch Kupfer überhaupt unerlässlich. Um dies bestimmt nachzuweisen, erbitzte ich gesăttigte Salmiaklösung und Kuplerspähne in einem verschlossenen Kolben, aus dem ein langes Rohr unter Wasser geleitet war, so dass kein Luftzutrilt staltinden konnte. Beim Erhitzen entwickelten sich, nachdem die atmosphärische Luft ausgetrieben war, nur noch Wasserdämpfe. Die Salmiaklüsung blieb während des ganzen Versucbs farblos. Zuletzt liess ich das Sperrwasser in den Kolben zurücksteigen und diesen vollständig erkalten. Es schied sich kein Kupferoxydul aus, doch hatte sich eine sehr kleine Henge weisser Krystalle gebildet, welche sich leicht als Kupferchlorür-Ammoniak erkennen liessen. Es hatte also ein Theil der dem Kupfer adhärirenden und in der Salmiaklösung befindlichen Luft hingereicht, um diese kleine Menge zu bilden. Die Reaction lässı sich, angenommen, dass Kupfer im Ueberschuss vorhanden ist, einfach so ausdrücken:

$$
2 \mathrm{Cu}+\mathrm{CINH}_{4}+\mathrm{O}=\mathrm{Cu}_{2} \mathrm{Cl}, \mathrm{NH}_{3}+\mathrm{HO} \text {. }
$$

Durch Autlösen dieses weissen Salzes in concentrirter Salzsăure und Erkalten hoffte ich den Kupferchlorür-Salmiak zu erhalten. Ich Jőste eine Partie weisser, oberflächlich etwas oxydirter Krystalle in möglichst wenig concentrirter heisser Salzsäure und verschloss das Glas. Nach dem Erkalten hatte die braune Lösung weisse Krystalle abgesetzt, welche sich an der Luft etwas bräunten, beim Erhitzen nur Salmiak, kein Ammoniak, gaben, mit Zurücklassung von Kupferchlorür. Sie zersetzten sich mit Wasser und wurden gelb; durch Alkohol wurden sie nicht verăndert. Die Analyse derselben ergab 48,44 p. C. Kupfer. Aus dieser einzigen Analyse, welche ich angestellt babe, würde sich die Formel berechnen:

welche 47,1 Kupfer verlangt.

$$
3 \mathrm{Cu}_{2} \mathrm{Cl}+2 \mathrm{CINH}_{4} \text {, }
$$

Ausserdem hatte sich eine ziemliche Menge KupferchloridSalmiak gebildet. Einige Versuche, die Verbindung $\mathrm{Cu}_{2} \mathrm{Cl}, \mathrm{CINH}_{4}$ darzustellen, waren erfolglos. 
Blaues Salz. Dieses Salz ist deswegen besonders interessant, weil es bei seinem hohen Gehalt an Kupferchlorür eine tief dunkelblaue Farbe besitzt. Dasselbe ist, wie aus den Analysen hervorgeht, eine Verbindung von Kupferchlorür-Ammoniak mit Kupferchlorid-Ammoniak und Wasser. Es entsteht aus der Flüssigkeit, welche Kupferchlorür-Ammoniak gelöst enthält, wenn diese mit Luft in Berührung kommt. Die Lösung wird schon während des Erhitzens beim Umrühren blau, beim Filtriren färbt sie sich noch dunkler. Aus sehr gesättigten Lösungen scheidet sich das Salz in langen dünnen aber gut ausgebildeten Prismen ab. Aus Lösungen, welche wenig davon enthalten, bildet es sich sehr langsam, aber dann in sehr schönen, regelmässig ausgebildeten grossen Krystallen*). Eben so erbält man sie sehr schōn, wenn man Salmiaklösung und Kupfer bei gewǒhnlicher Temperatur lange auf einander einwirken lässt; hierbei entsteht nur eine sehr kleine Menge Kupferchlorũr-Ammoniak, welches sehr fest am Kupfer haftet. Die Haufwerke der blauen Krystalle, welche sich aus sehr gesältigten Lösungen ausscheiden und aus langen aber dünnen Prismen bestehen, zersetzen sich an der Luft sehr schnell, sie überziehen sich mit einer bläulich-grūnen Schicht und verlieren dann allen Glanz; die Ursache hiervon liegt besonders darin, dass sie von der anhängenden Multerlauge nicht befreit werden können; Pressen zwischen Fliesspapier hilft nicht viel. Durch Wasser und Alkohol werden sie zersetzt, in Salmiaklősung lősen sie sich ziemlich bedeutend und werden durch dieselbe auch theilweise zersetzt. $\mathrm{Zu}$ den Analysen benutzte ich besonders die grōssern Krystalle, welche ich einzeln mit Fliesspapier trocknete. Gut getrocknet sind die Krystalle an der Luft ziemlich beständig, doch werden sie immer matt und nach längerer Zeit auch oberflächlich zersetzt durch Verlust von Wasser, Ammoniak und Absorption von Sauerstoff.

Die Krystallform des Salzes konnte durch Messung nicht

*) Die sehönsten Krystalle erhielt ich in- zwei Versuchen, in denen ich kalt gesāttigte Salmiaklơsung angewendet hatte. Es war nur wenig Kupfer aufgelost worden; die Flüssigkeit war aber ziemlich blau. Nach länger als 24 Stunden batten sich bei starker Abkühlung über $11 / 2$ Cent. lange und mehrere Millimeter dicke Krystalle gebildet, ron ganz regelmässigen Formen. 
bestimmt werden; die Grősse und regelmässige Ausbildung der Krystalle aber erlaubte, dieselben approximativ zu hestimmen. Sie scheinen rhombisch zu sein; sie bilden grade vierseitige Prismen, welche mit einer vierllächigen Pyramide, deren Flächen über den Kanten des Prismas liegen, combinirt sind; sehr häufig sind die Prismenkanten parweise oder auch alle abgestumpft, eben so die Polkanten. Uebrigens warèn einzelne der grössten Krystalle inwendig hohl und an den innern Seiten dann auch zersetzt.

Das Salz wird von Wasser und Alkohol zersetzt. Wird es mit Wasser gekocht, so scheidet sich Kupferoxydul und ein gelbgrünes Pulver ab, wāhrend yom Wasser Salmiak und Ammoniak ausgezogen werden. In Säuren ist es ohne Zersetzung nicht löslich. An der Luft geglültt giebt es etwas Wasser, Salmiak, Ammoniak und als Rückstand Kupferchlorür. Bei $100^{\circ}$ getrocknet verliert es Wasser und vielleicht auch Spuren von Ammoniak; die Krystalle werden dann dunkelbraun. Das Pulver frischer Krystalle ist violett, wird aber nach längerem Stehen an der Luft grünlich.

Die Analysen wurden wie die des Kupferchlorür-Ammoniaks ausgeführt. Das Wasser bestimmte ich aus dem Verlust. Es wurden aber Produkte von verschiedenen Bereitungen analysirt.

Erhalten wurden folgende Resultate:

$0,630 \mathrm{Grm}$. Salz gab. $\mathrm{CuO}=0,370 \mathrm{Grm} .=0,295 \mathrm{Cu}=45,38 \mathrm{p}$. C. $0,748, ", "=0,4305,=0,344,=46,01$, $1,151, ", ", \quad "=0,657 \quad,=0,524,=45,48$ " $0,2835 ", " \quad \mathrm{ClAg}=0,387, \quad=0,096 \mathrm{Cl}=34,21$ " $0,437, "$ " Pt $=0,4325,=0,074 \mathrm{NH}_{3}=16,93$ ", $0,385 ", ", "=0,36 \tau, "=0,063,=16,37$ "

Die Mittelzahlen sind:

$$
\begin{aligned}
& \mathrm{Cu}=45,62 \\
& \mathrm{Cl}=34,21 \\
& \mathrm{NH}_{3}=16,65
\end{aligned}
$$

Daraus lässt sich die Formel berechnen:

$$
\mathrm{Cu}_{2} \mathrm{Cl}, \mathrm{NH}_{3}+\mathrm{CuCl}, \mathrm{NH}_{3}+\mathrm{HO}
$$

welche verlangt: 


$$
\begin{aligned}
& \mathrm{Cu}=45,54 \\
& \mathrm{Cl}=33,90 \\
& \mathrm{NH}_{3}=16,26 \\
& \mathrm{HO}=4,3
\end{aligned}
$$

Beim vorsichtigen Trocknen unter $100^{\circ}$ gaben $0,5625 \mathrm{Grm}$. Substanz 0,020 Wasser, also 3,5 p. C.

Die Bildung dieses Salzes ist leicht zu erklären. Wenn die Kupferchlorūr-Ammoniak enthaltende Lösung vom Kupfer abgegossen, also eine weitere Aufnahme von diesem verhindert, dagegen aber der Zutritt von Sauerstoff nicht gehindert wird, so nimmt die Lösung Sauerstoff auf; es bildet sich etwas Kupferchlorid und Kupferoxyd, und durch Zersetzung dieses letztern mit Salmiak wieder Kupferchlorid-Ammoniak, welches mit Salmiak verbunden in Lösung bleibt, und Wasser. Die Reaction lässı sich etwa durch folgende Gleichung ausdrücken:

$$
\begin{aligned}
& 2 \mathrm{Cu} \mathrm{Cl}_{2} \mathrm{NH}_{3}+\mathrm{O}=\mathrm{Cu} \mathrm{Cl}_{3} \mathrm{NH}_{3}+\mathrm{CuCl}, \mathrm{NH}_{3}+\mathrm{CuO} . \\
& \mathrm{CuO}+2 \mathrm{ClNH}_{4}=\mathrm{CuCl}, \mathrm{NH}_{3}+\mathrm{ClNH}_{4}+\mathrm{HO} .
\end{aligned}
$$

Die Formel $\mathrm{CuCl}, \mathrm{NH}_{3}+\mathrm{ClH}_{4} \mathrm{~N}$ ist in der That die Formel des im Eingange erwăhnten schōn grünen Salzes.

Löst man das dunkelblatle Salz in Salzsäure oder Salpetersàure in der Wärne auf, so entsteht die Verbindung $\mathrm{CuCl}, \mathrm{CJNH}_{4}$ $+2 \mathrm{HO}$, welche sich nach dem Erkalten in schönen hellblauen Krystallen absetzt. Dieses Salz ist von Mitscherlich, Cap und Henry, Vogel etc. schon genügend untersucbt, so dass ich mich auf die Resultate der Analyse beschränke.

Ber.

Gef.

$$
\begin{aligned}
& \mathrm{Cu}=31,7 \quad 22,8 6 \quad \longdiv { 2 3 , 5 2 \text { p. C. } 2 3 , 1 9 } \\
& 2 \mathrm{Cl}=71,0 \quad 51,20 \quad 50,80 \quad, \quad- \\
& \mathrm{NH}_{4}=18,0 \quad 12,97 \quad 12,67 \quad, \quad- \\
& 2 \mathrm{HO}=18,0 \quad 12,97 \quad-\quad
\end{aligned}
$$

Grünes Salz. Dieses Salz ist durch seine schơn grüne Farbe ausgezeichnet. Es existirt fertig gebildet in der Mutterlauge von den blauen Krystallen, wie schon gezeigt worden ist, es bildet sich aber auch noch weiter, wenn man die Mutterlauge an der Luft stehen lässt; diese färbt sich von oben herein grün, so dass sie zwei Schichten zeigt, von denen die untere blall, die obere schőn grün ist; allmählich wird sie ganz grün. Sal- 
miaklösungen, welche mehrere Male zur Darstellung des blauen Salzes angewendet worden sind, enthalten schon ziemlich viel vom grünen Salze in Auflösung, so dass sie bläulich-grūn aussehen und nach kurzer Zeit eine Menge Krystalle absetzen. Aus solchen gesättigten Lüsungen kann man das Salz in ziemlich grossen, schŏn ausgebildeten Würfeln crhalten, wenn man bei niedriger Temperatur krystallisiren lässt. Gewöhnlich scheidet es sich in kleinen Würfeln $a b$, welche auf der Oberfläche sch:vimmen.

Die grőssern Krystalle sind dunkelgrün, äusserst glänzend, die kleinern lebhaft grün und ganz durchsichtig, alle aber sind regelmāssig ausgebildete Würfel. Durch Behandlung mit Wasser werden sie in ein blaues, später gelblich-grünes Pulver verwandelt. Das Wasser löst etwas Chlorkupfer, zieht Ammoniak und Salmiak aus; Alkohol wirkt ähulich, aber in geringerem Grade, so dass sie mit kaltem Alkohol unbedenklich gewaschen werden kŏnnen. In Säuren sind sie ohne Zersetzung nicht autlōslich. Bei $100^{\circ}$ verlieren sie gar nichts an Gewicht und bleiben vollkommen unverändert. Geglüht entwickeln sie Ammoniak, Salmiak und lassen, als Rückstand Kupferchlorūr.

Die Analyse ausgesuchter und von anhängendem Salmiak ganz freier Krystalle des Salzes ergab folgende Zahlen:

$0,4575 \mathrm{Grm}$. gaben $\mathrm{CuO}=0,137 \mathrm{Grm} .=0,109 \mathrm{Cu}=23,82 \mathrm{p}$. C.

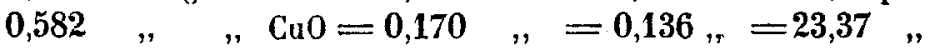
$0,3075 " \quad " \mathrm{ClAg}=0,6305, "=0,156 \mathrm{Cl}=50,73 "$ $0,3345 " \quad$ "Pt $=0,460 ",=0,080 \mathrm{NH}_{3}=23,916 "$

Aus diesem lässt sich die Formel ableiten:

welche verlangt:

$$
\mathrm{CuCl}, \mathrm{NH}_{3}+\mathrm{CINH}_{4} \text {, }
$$

$$
\begin{array}{cr}
\mathrm{Cu} & =23,02 \\
2 \mathrm{CI} & =51,56 \\
\mathrm{NH}_{3}+\mathrm{NH}_{4} & =25,42
\end{array}
$$

In verdünnter heisser Salzsäure gelöst giebt das Salz Chlorkupfer-Salmiak, wie das vorige, eben so, wenn es in verdünnter Salpetersāure gelōst wird.

Die Kupfersalze, welche als Produkte der Einwirkung von Salmiak, Kupfer und Luft gefunden wurden, sind: 
Kupferchlorür-Ammoniak

Kupferchlorid-Ammoniak $=\mathrm{Cu}_{2} \mathrm{Cl}, \mathrm{NH}_{3}$. $=\mathrm{Cu}_{2} \mathrm{Cl}, \mathrm{NH}_{3}+\mathrm{CuCl}, \mathrm{NH}_{3}+\mathrm{HO}$ Kupferchlorid-Ammoniak-Salmiak $=\mathrm{Cul}_{2} \mathrm{Cl}, \mathrm{NH}_{3}+\mathrm{ClNH}_{4}$.

Die bei der ersten Einwirkung gebildeten und auf der Oberfläche schwimmenden Krystallnadeln zeigten sich bei der Prüfung als etwas chlorkupferhaltiger Salmiak.

Veber die Entstehung des Braunschweiger Grüns. Es gilt nun nachzuweisen, ob und auf welche Art sich aus diesen Verbindungen das Braunschweiger Grün darstellen lässt. Besonders wichtig hierbei schien mir das blaue Salz und die blaue Kruste, welche sich bei jeder Behandlung des Kupfers mit Salmiak bildet; sie musste nothwendig ein Zersetzungsprodukt aus dem dunkelblauen Salze sein, denn dieses lieferte in Salmiak gelöst und anhaltend gekocht, wobei viel Ammoniak entwich, ebenfalls ein blaues Pulver. Lüsst man ersteres nach der Bereitung mit der anhängenden Mutterlauge an der Luft nur einige Zeit stehen, so giebt es ein hellblaues Pulver. In allen den genannten Fällen verwandelt sich das Kupferchlorür des Salzes in Kupferchlorid und Kupferoxyd. Wird nun dieses blaue Pulver, auf welche Weise es auch erbalten sein mag, init heissem Wasser ausgelaugt, so liefert es zuletzt ein blau-grūnes Pulver von dem Ansehen des Braunschweiger Grüns. Das Wasser löst Chlorkupfer, Ammoniak und Salmiak aul, doch sind nur die ersten Wässer stark blau gefärbt. Ich setzte die Auswaschung mit heissem Wasser so lange fort, bis ich eine nur sehr schwache Reaction auf Chlor erhielt; dann wurde das Pulver getrocknet und analysirt. Zur Vergleichung habe ich das Produkt aus den blaten Krusten und das aus dem zerselzten dunkelblauen Salze besonders analysirt.

Das weisse Salz erzeugt, wenn es gerieben mit etwas Wasser angefeuchtet wird, durch Oxydation eine blaugrüne Verbindung, aus welcher Wasser Ammoniak, Salmiak und Kupferchlorid auszieht, wăhrend eine Substanz, ganz von dem Ansehen des Braunschweiger Grüns, zurückbleibt; ich babe sie nicht analysirt. Ein āhnliches Produkt erhält man aus dem grünen Salze durch Behandlung mit Wasser, bis die Reaction auf Chlor kaum bemerkbar ist. -

Die Analysen der beiden Grün, welche $I$. aus den blauen 
Krusten, II. aus den verwitterten blauen Krystallen dargestellt waren, führten zu folgenden Resultaten:

I. Lufttrocken.

$0,977 \mathrm{Grm}$. gaben $0,5158 \mathrm{CuO}=0,412 \mathrm{Cu}=42,17 \mathrm{p}$. C.

$0,310 \quad, \quad, \quad 0,169 \quad \mathrm{ClAg}=0,042 \mathrm{Cl}=13,55 \quad$,

Bei $100^{\circ}$ getrocknet.

$0,4175 \mathrm{Grm}$. wogen nach mehrstündigem Trocknen 0,378 Grm., sie hatten also 0,0395 Wasser verloren $=9,48$ p. C.

Diese 0,378 Grm. gaben $\mathrm{CuO}=0,287=0,229 \mathrm{Cu}=$ 60,58 p. C.

$0,168 \mathrm{Grm}$. ebenfalls bei $100^{\circ}$ getrocknet gaben ClAg 0,099 $=0,024 \mathrm{Cl}$, also 14,28 p. C.

II. Luftrocken.

1,0345 Grm. gaben $0,774 \mathrm{CuO}=0,567 \mathrm{Cu}=54,81$ p. C. $0,4735 \quad " \quad 0,296 \mathrm{ClAg}=0,080 \mathrm{Cl}=16,9$ ",

Bei $100^{\circ}$ getrocknet.

$1,1955 \mathrm{Grm}$. verloren 0,0235 , also 1,9 p. C. an Gewicht.

$0,832 \mathrm{Grm}$. dieser getrockneten Substanz gaben $0,631 \mathrm{CuO}$ $=0,511 \mathrm{Cu}$ oder $61,418 \mathrm{p}$. C.

$0,340 \mathrm{Grm}$. dieser getrockneten Substanz gaben 0,2135 ClAg $=0,053 \mathrm{Cl}$, also $15,6 \mathrm{p}$. C.

Aus diesen Zahlen berechnet sich für $\mathrm{I}$.:

Nicht getrocknet $\mathrm{CuCl}=25,65$ p. C.

$\mathrm{CuO}=37,60$,

$\mathrm{HO}=36,75$,

Getrocknet

$\mathrm{CuCl}=27,03 \quad "$

$\mathrm{CuO}=59,9$

HO $=13,07$,

Für II.:

Nicht getrocknet $\mathrm{CuCl}=31,99$ p. C.

$\mathrm{CuO}=49,744$,

$\mathrm{HO}=18,266$,

Getrocknet

$\mathrm{CuCl}=29,53 \quad$ "

$\mathrm{CuO}=59,6 \quad$,

$\mathrm{HO}=10,87$ " 
Diese Resultate entsprechen den Formeln:
I. Nicht getrocknet $\mathrm{CuCl}+3 \mathrm{CuO}+10 \mathrm{HO}$. Getrocknet
$\mathrm{CuCl}+3 \mathrm{CuO}+3 \mathrm{HO}$.
II. Nicht getrocknet $\mathrm{CuCl}+3 \mathrm{CuO}+4 \mathrm{HO}$. Getrocknet
$\mathrm{CuCl}+3 \mathrm{CuO}+3 \mathrm{HO}$.

Auf die Formeln der nicht getrockneten Verbindung ist nach neinem Dafürhalten kein Werth zu legen, da man nach kürzerem oder längerem Trucknen verschiedene Mengen Wasser finden wird; doch ist das Verhältniss von $\mathrm{CuCl}: \mathrm{CuO}=1: 3$ constant. Nach den Analysen von Proust hat man dem Körper allgemein die Formel $\mathrm{CuCl}, 3 \mathrm{CuO}+4 \mathrm{HO}$ gegeben.

Die Bildung des hellblauen Pulvers, aus welchem das Grün durch Auswascben entsteht, aus den blauen Krystallen lässt sich einfach durch die Gleichung ausdrücken:

$$
\begin{gathered}
\mathrm{Cu}_{2} \mathrm{Cl}, \mathrm{NH}_{3}+\mathrm{CuCl}, \mathrm{NH}_{3}+\mathrm{HO}+\mathrm{O}=2\left(\mathrm{CuCl}, \mathrm{NH}_{3}\right) \\
+\mathrm{CuO}+\mathrm{HO} .
\end{gathered}
$$

Die Zersetzung dieses Pulvers durch Waschen mit Wasser in Braunsch ıveiger Grün lässt sich schwerlich durch eine einfache Gleichung bezeichnen.

\title{
XLIV.
}

\section{Ueber das Didym und seine wichtigsten Verbindungen.}

\author{
Von \\ C. Mrarignac.
}

(Ann. de chim. et de phys. 3. Ser. XXXV111, 148.)

Seit der 1841 von Mosander gemachten Entdeckung des Didyms im Cerit ist keine wichtige Arbeit über die Verbindungen dieses Metalls erschienen, und unsre Kenntnisse von demselben beschränken sich fast nur auf die sebr kurzen Angaben, welche der Entdecker selbst gegeben hat. H. Watts*) veröffentlichte

-) Quart. Journ. of the Chem. Soc. of London, II. 140. 